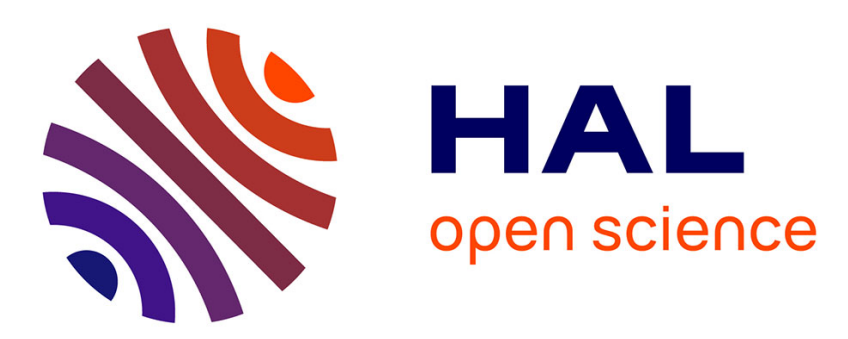

\title{
Une religion politique. Les usages des martyrs révolutionnaires dans le royaume des Deux-Siciles (années 1820-années 1850)
}

Pierre-Marie Delpu

\section{- To cite this version:}

Pierre-Marie Delpu. Une religion politique. Les usages des martyrs révolutionnaires dans le royaume des Deux-Siciles (années 1820-années 1850). Revue d'Histoire Moderne et Contemporaine, 2017, 64 (1), pp.7-31. halshs-01537675

\section{HAL Id: halshs-01537675 \\ https://shs.hal.science/halshs-01537675}

Submitted on 12 Jun 2017

HAL is a multi-disciplinary open access archive for the deposit and dissemination of scientific research documents, whether they are published or not. The documents may come from teaching and research institutions in France or abroad, or from public or private research centers.
L'archive ouverte pluridisciplinaire HAL, est destinée au dépôt et à la diffusion de documents scientifiques de niveau recherche, publiés ou non, émanant des établissements d'enseignement et de recherche français ou étrangers, des laboratoires publics ou privés. 
Revue d'histoire moderne et contemporaine, 64-1, janvier-mars 2017, pp. 7-31.

\title{
Une religion politique. Les usages des martyrs révolutionnaires dans le royaume des Deux-Siciles (années 1820-années 1850)
}

\author{
Pierre-Marie Delpu
}

\begin{abstract}
«Dans les provinces méridionales, continentales comme insulaires, le martyre politique est une tradition : au lieu de provoquer l'effroi, il a toujours généré des héros, lesquels, placés à leur tour sous le joug du pouvoir, en ont produit d'autres, et ces derniers, une fois soumis, ont fini par créer une conscience et une opinion publique d'opposition, afin qu'elle serve ensuite de base à la révolution morale puis à la révolution matérielle à venir ${ }^{1}$.
\end{abstract}

Dans cette chronique rédigée sept ans après l'annexion du royaume des Deux-Siciles à l'Italie unie, le démocrate napolitain Giuseppe Lazzaro (1825-1910) évoque la pratique méridionale du martyrologe politique, dans laquelle il voit l'un des éléments constitutifs de l'identité culturelle méridionale. Construite par des généalogies fictives entretenues par les acteurs, elle implique plusieurs générations de patriotes qui ont en commun de s'être opposés à l'absolutisme et à la violence politique exercées par l'armée et la police bourboniennes, qui ont réprimé plusieurs révoltes et révolutions locales ou nationales ${ }^{2}$. Les martyrs ne sont ni nommés ni datés, précisément parce qu'ils témoignent d'un caractère intemporel de la nation napolitaine. Ce que l'auteur qualifie de «martyre » à la suite d'une importante tradition méridionale est toutefois dépourvu d'un sens précis : sa connotation religieuse est claire, impliquant le sacrifice personnel, mais ses échos politiques sont très divers, englobant les privations, les souffrances, l'exposition à la violence politique mais aussi l'exil, vécu comme une souffrance psychologique produite par l'éloignement de la patrie.

Dans l'opposition libérale aux Bourbons, les usages des martyrs ont revêtu un double aspect. De très nombreux martyrologes littéraires ont en effet été publiés, dont les plus célèbres sont ceux du modéré Mariano D’Ayala (1808-1877) au moment de l'unification italienne $^{3}$. Ces écrits sont les héritiers des développements locaux du romantisme, qui a connu

1. Giuseppe LAZZARO, Memorie della rivoluzione dell'Italia meridionale dal 1848 al 7 settembre 1860, Naples, Stabilimento tipografico dei classici italiani, 1867, p. 140-141. Sauf mention contraire, les traductions de l'italien sont les nôtres.

2. À l'échelle nationale, la police et l'armée ont notamment réprimé les révolutions de 1799, de 1820-21 et de 1848. De manière plus ponctuelle et localisée, elles ont mis fin aux révoltes rurales dans les régions périphériques du Cilento (1828 et 1848), des Abruzzes (1837 et 1841) et des Calabres (1837, 1844 et 1848).

3. Mariano D'Ayala commence, dans les années 1850, à produire des écrits divers avec l'ambition de construire un martyrologe national. L'œuvre la plus célèbre demeure Vite degl'Italiani benemeriti della libertà e della patria, morti combattendo, Florence, Cellini, 1868, dont les premières pages sont écrites dans les années 1850 , en exil à Turin. 
dans le royaume l'une de ses deux seules écoles italiennes avec la Lombardie ${ }^{4}$. Ils témoignent de registres spécifiques d'émotivité qui ont influencé de façon déterminante les pratiques politiques, reflétant l'aspect sentimental, viscéral et presque religieux du patriotisme libéral, conçu comme une « religion de la liberté $»^{5}$. La construction des mémoires révolutionnaires, la référence constante aux héros fondateurs, les usages sociaux de la mort politique en ont été les manifestations principales. D'autre part, la mémoire des martyrs révolutionnaires a été l'un des principaux supports de la politisation populaire à l'échelle locale. Cette dernière, entendue comme la «pénétration de la politique » auprès des masses du royaume ${ }^{6}$, constitue l'un des aspects essentiels d'un mouvement plus large, l'entrée en modernité politique, dont les ressorts sont désormais connus pour l'Italie et l'espace méditerranéen au sens large ${ }^{7}$. Alors qu'elle s'est la plupart du temps accompagnée d'un relatif «apprentissage » de la politique, elle a reposé, dans le royaume des Deux-Siciles, sur des réflexes spontanés, très largement empruntés aux pratiques sociales ordinaires, dans un contexte désormais réenvisagé comme une guerre civile permanente ${ }^{8}$. Le religieux en a fait partie, soit parce que ses acteurs ont été des appuis ou des opposants des révolutions, soit parce qu'il a fourni des cadres mentaux permettant de concevoir des réalités très largement méconnues du peuple.

Les provinces calabraises offrent de ce point de vue un observatoire particulier. Très bien documentées dans les archives napolitaines et locales, elles fournissent les exemples les plus nombreux de cultes des martyrs politiques, principalement autour du moment 1848 qui en est l'un des temps forts. L'exploitation politique des figures de martyrs doit néanmoins être envisagée dans un temps plus long, de ses premières manifestations dans la révolution de 1820-21 à la multiplication des martyrologes littéraires au moment de l'unification italienne. Elle a contribué à former l'identité culturelle du patriotisme libéral napolitain, auquel se

4. Sur le romantisme calabrais, voir notamment Gaetano CINGARI, Domenico Mauro. Democrazia e romanticismo nel Mezzogiorno, Lungro, Marco, 2001.

5. Nous empruntons l'expression à Benedetto CROCE, Histoire de l'Europe au XIXe siècle [1932], Paris, Gallimard, 1959, p. 43.

6. Nous reprenons ici la définition proposée par Maurice AGULHON, «Présentation », in COLLECTIF, La politisation des campagnes au XIX ${ }^{e}$ siècle. France, Italie, Espagne et Portugal, Rome, École française de Rome, 2000, p. 2-11.

7. Les prolongements italiens des travaux de Maurice Agulhon ont été de ce point de vue déterminants. Voir en particulier Franco RIZZI, La coccarda e le campane : comunità rurali e Repubblica romana nel Lazio 18481849, Milan, FrancoAngeli, 1988 ou, sur la Calabre, d’Antonio ButTiGLionE, « "Revindiche". Violenza sociale e mobilitazione politica in Calabria nel $1848 »$, Aiônos. Miscellanea di studi storici, 18, 2014, pp. 135-174.

8. Carmine PINTO, «Tempo di guerra. Conflitti, patriottismi e tradizioni politiche nel Mezzogiorno d'Italia (1859-1866) », Meridiana. Storia e scienze sociali, 76, 2013, p. 57-84. Le cas de la Calabre est le mieux couvert par la bibliographie, y compris internationale : voir par exemple Domenico CECERE, « Contre les "tyrans". Luttes judiciaires et troubles anti-seigneuriaux en Calabre au XVIII ${ }^{\mathrm{e}}$ siècle", Revue d'histoire moderne et contemporaine, 60, 2013/3, p. 7-30 ; Nicolas CADET, Honneur et violences de guerre au temps de Napoléon. La campagne de Calabre, Paris, Vendémiaire, 2015. 
superpose progressivement, à partir des années 1830, une identité péninsulaire italienne au contenu variable ${ }^{9}$. La valeur de politisation des martyrs révolutionnaires a été sous-évaluée par l'historiographie, qui les a réduit à un aspect parmi d'autres de la mémoire politique de la révolution de 1799, qui aurait influencé la pensée et l'action des élites libérales du Risorgimento $^{10}$. Les usages politiques de la mort à l'époque romantique sont connus ${ }^{11}$, mais c'est plutôt dans l'histoire des guerres de Religion de l'époque moderne qu'il faut rechercher le processus de formation sociale des martyrs et leurs usages dans les comportements politiques quotidiens, informels et locaux. Les travaux de David El Kenz ont notamment montré comment, à côté des martyrs canoniques, s'étaient formés des figures locales de martyrs, nourrissant une culture politique de la désobéissance et de la transgression capable de mobiliser les masses ${ }^{12}$. Le martyre révolutionnaire du XIX ${ }^{\mathrm{e}}$ siècle nous semble obéir à des logiques comparables. On postulera que le royaume des Deux-Siciles a vu se former une culture libérale du martyre qui a servi à la politisation locale en même temps qu'elle a été l'un des supports de l'identité de la nation napolitaine, à l'heure de la construction nationale italienne. Emprunté au registre religieux, le martyrologe libéral s'est développé en tant que tradition littéraire dès le début du $\mathrm{XIX}^{\mathrm{e}}$ siècle, en s'appuyant sur les mémoires croisées des révolutions successives connues par le royaume. Le «moment 1848 » a vu s'amplifier le phénomène à travers la célébration des martyrs locaux, à la fois l'objet de rituels commémoratifs et de réflexes de vengeance visant à expier des massacres d'État opérés par les forces de l'ordre. La Calabre en offre les cas les plus significatifs à travers les fusillés de Cosenza (1844) et de Gerace (1847). Après la répression de la révolution de 1848, les martyrs libéraux font l'objet d'usages mémoriels contrastés, entre intégration au panthéon national italien et valorisation de l'identité napolitaine.

9. Principalement Giuseppe MASSARI, I casi di Napoli dal 29 gennaio 1849 in poi, Turin, Vecchi, 1849. Sur la nation napolitaine, voir Aurelio MUSI, «La nazione napoletana prima della nazione italiana », in Angela DE BENEDICTIS, Irene FOSI, Luca MANNORI (éd.), Nazioni d'Italia. Identità politiche e appartenenze regionali fra Settecento e Ottocento, Rome, Viella, 2012, p. 75-90.

10. Renata DE LORENZO, «La tradizione del 1799 nel Risorgimento italiano », in EAD., Un regno in bilico. Uomini, eventi e luoghi nel Mezzogiorno preunitario, Rome, Carocci, 2001, p. 357-371.

11. La principale étude, pour la France, est celle d'Emmanuel FUREIX, La France des larmes. Deuils politiques à l'âge romantique 1814-1840, Seyssel, Champ Vallon, 2009.

12. Notamment David El KenZ, Les bûchers du roi. La culture protestante des martyrs 1523-1572, Seyssel, Champ Vallon, 1997. 
DU RELIGIEUX AU POLITIQUe, NAISSANCE D'UNE TRADITION DU MARTYROLOGE (18201848)

L'échec chronique des révolutions et le souvenir des victimes qui se sont sacrifiées pour la liberté et pour la patrie constituent des thèmes discursifs forts, des lieux de mémoire communs à l'opposition aux Bourbons. Les figures de martyrs libéraux ou révolutionnaires, dont le culte se développe dans les premières décennies du $\mathrm{XIX}^{\mathrm{e}}$ siècle, cristallisent des identités politiques d'opposition encore mouvantes et imprécises entre les liberali, modérés, héritiers des Lumières et de la culture politique de la Révolution française, et les democratici, plus radicaux et très majoritairement républicains ${ }^{13}$.

La catégorie martyr relève au départ du lexique religieux et a connu ses premiers développements politiques à propos des victimes de la révolution de 1799. Celles-ci sont évoquées en 1800 par le Saggio storico de Vincenzo Cuoco, perçu par les élites libérales méridionales comme un texte fondateur, en grande partie parce qu'il évoque une situation longtemps perçue comme traumatique ${ }^{14}$. L'attestation est néanmoins restée isolée, à l'exception de quelques textes très ponctuels publiés par des patriotes en exil en France au lendemain de la révolution ${ }^{15}$. C'est surtout dans les années 1820 que la référence aux martyrs s'impose comme un lieu commun des discours des libéraux : lorsque la révolution de 1820-21 est menacée par l'armée autrichienne aux frontières du royaume, venue rétablir l'ordre voulu par la Sainte-Alliance, le député modéré Giuseppe Poerio (1775-1843) rappelle, au cours d'une session parlementaire, la mémoire des patriotes de 1799, morts pour la liberté et la révolution, faisant référence à leur «mépris généreux de la mort qui fit un temps les martyrs de la Religion et qui fait, aujourd'hui, les martyrs de la liberté [souligné par nous] ${ }^{16}$. L'invocation des patriotes morts pour la liberté permet d'appeler à la constance et à la mobilisation pour la patrie en danger: leur ancestralité, leurs valeurs d'humanité, leur

13. On appellera, en suivant la terminologie utilisée par les acteurs, liberali les héritiers des Lumières napolitaines, des théories fondatrices de Vincenzo Cuoco sur la révolution de 1799 et du réformisme du decennio francese. Le terme, utilisé au départ dans les pratiques d'auto-désignation des révolutionnaires, est ensuite récupéré par le langage administratif et policier des Bourbons. Voir Pierre-Marie DELPU, «Fraternités libérales et insurrections nationales : Naples et l'Espagne, 1820-1821 », Revue d'histoire du XIX ${ }^{e}$ siècle, 49, 2014/2, p. 193-211. La distinction entre liberali et democratici, que ne pratiquent pas les sources bourboniennes, tend à se préciser à partir des années 1830 .

14. Sur la fortune des écrits de Vincenzo Cuoco pendant la Restauration : Raffaele PITTELLA, «Il rilievo dell'opera di Cuoco nella cultura politica del Mezzogiorno napoleonico, 1806-1821», in Luigi BISCARDI, Antonino De FrAnCESCo (éd.), Vincenzo Cuoco nella cultura politica di due secoli, Rome-Bari, Laterza, 2002, p. 151-161. Voir également Vincenzo CuOCO, Saggio storico sulla rivoluzione di Napoli, édition d'Antonino De Francesco, Rome-Manduria, Lacaita, 1998.

15. Anna Maria Rao en a relevé plusieurs attestations dans les écrits des patriotes napolitains exilés en France après 1799. Voir Esuli. L'emigrazione politica italiana in Francia (1792-1802), Naples, Guida, 1992, p. $362-369$ et 417-421.

16. Giornale Costituzionale del Regno delle Due Sicilie, 52, $1^{\mathrm{er}}$ mars 1821, p. 3. 
pratique commune du sacrifice personnel permettent de les constituer en figures morales exemplaires. La référence religieuse témoigne de la sacralité de ces martyrs, évoquant les figures saintes de l'Antiquité tardive, destinées à susciter l'admiration et l'enthousiasme, à constituer des exemples de moralité ${ }^{17}$. Sa signification sociale et culturelle apparaît évidente dans une société où la très forte religiosité populaire a été l'un des instruments principaux de la mobilisation politique, qu'elle soit libérale ou contre-révolutionnaire. Majoritairement réactionnaire en 1799, le clergé méridional a par la suite constitué l'un des soutiens principaux des révolutions politiques. En 1820-21 en effet, les curés de village ont contribué de manière déterminante à la diffusion des idées libérales, exploitant leur double fonction de prédicateurs et d'enseignants, essentiellement dans les zones rurales des provinces calabraises et des deux Principats (carte 1). Ils ont en même temps été fortement représentés parmi les dignitaires des sociétés secrètes, précisément parce qu'ils étaient très souvent les seuls à pouvoir en comprendre et en interpréter le message, dans une société très majoritairement analphabète et dialectophone. Ce rôle de politisation des prêtres s'est retrouvé dans la totalité du mouvement libéral, y compris dans sa frange la plus radicale. L'alliance entre les sphères religieuse et politique, constante jusqu'à l'Unité italienne, s'est néanmoins très largement limitée au bas-clergé et s'est fondée sur une culture politique catholique : la constitution espagnole de 1812, adoptée par les révolutionnaires napolitains de 1820-21, définissait le royaume comme une nazione cattolica, envisageant la religion comme le ciment social de la communauté politique. Cette conception s'est exprimée dans l'ensemble des pays héritiers de la Contre-réforme qui ont suivi le modèle libéral espagnol, où elle s'est appuyée sur le rôle du clergé $^{18}$. Leur implication a été particulièrement massive dans le royaume des Deux-Siciles où la prédication a servi de modèle à l'intermédiation politique et où le religieux a très largement modelé la compréhension des réalités politiques.

La perception du martyre politique est tributaire de ces conceptions, expliquant qu'elle ne soit que très rarement explicitée. Parmi les ouvrages publiés dans la capitale du royaume, la thématique du martyre est courante et relève, jusqu'aux années 1840, essentiellement de l'histoire sainte. Les attestations de martyrs napolitains y sont nombreuses, à commencer par saint Janvier, protecteur de la capitale du royaume. Transposé dans l'ordre politique, le terme martyr fait référence à tous les opposants qui ont subi, à des degrés divers, l'oppression

17. Jean-Pierre ALBERT, «Sens et enjeux du martyre : de la religion à la politique », in Pierre CENTLIVRES (éd.), Saints, sainteté et martyre. La fabrique de l'exemplarité, Neuchâtel, Éditions de l'Institut d'Ethnologie, 2001, p. 17-26.

18. Maurizio IsABELLA, «Citizens or faithful ? Religion and the liberal revolutions of the 1820s in Southern Europe », Modern Intellectual History, 2015/1, p. 1-40. 
politique exercée par la monarchie bourbonienne. Cela implique une vision romantique de la politique fondée sur le sacrifice personnel et sur l'héroïsation, sans que la référence à la mort ou à la mutilation corporelle ne soit nécessaire. Définir les patriotes napolitains comme des martyrs revient donc à définir le sacrifice comme un élément identitaire, les rattachant à la culture politique méridionale en tant qu'héritiers du saint patron de Naples. Cette lecture, largement pratiquée par les sociétés secrètes libérales qui placent le martyre au centre de leur iconographie $^{19}$, voit dans le sacrifice héroïque au nom de la souveraineté populaire une manifestation de la vertu politique; elle trouve ses prolongements dans les très nombreuses histoires de Naples que publient les exilés libéraux après l'échec de la révolution de 1820-21. Un libéral comme Guglielmo Pepe, dans ses très nombreux écrits à prétention historique, mais aussi des auteurs plus modérés comme Pietro Colletta ou Luigi Blanch brossent le portrait d'une nation-martyr qui a souffert, depuis le Moyen Âge, de la réaction politique et de la coercition exercées d'abord par les occupants étrangers, barbares, français ou espagnols, puis par les Bourbons, à la tête d'une monarchie indépendante depuis 1734, alors que les Napolitains auraient œuvré de façon constante pour leur liberté. Ces écrits établissent des généalogies fictives entre la révolte de Masaniello de 1648, les conspirations jacobines des années 1790, les révolutions de 1799 et de 1820-21. Cette parenté se fonde sur l'intérêt commun que les Méridionaux auraient porté à la liberté de leur patrie et sur les caractéristiques morales communes attribuées aux combattants qui y ont péri. Dans un opuscule écrit en exil à Paris en 1840, Pepe rappelle l'oppression permanente exercée par les Bourbons contre «les hommes les plus distingués par leur talent, par leur richesse, par leur naissance », systématiquement condamnés à la prison ou à l'exil, situation dont les condamnés de 1799 lui semblent constituer le cas le plus significatif. Il évoque ainsi le philosophe Mario Pagano (1748-1799), mort pour satisfaire le peuple ${ }^{20}$ et, dans un autre texte, le jeune soldat Ettore Carafa (1763-1799) dont il souligne le courage, l' « amour de la patrie » et 1 ' «ambition immodérée $»^{21}$. Ces personnages, qui ont en commun d'être nobles, vertueux et patriotes, servent à incarner l'histoire napolitaine marquée par les mémoires croisées des révolutions successives qui constituent une culture politique complexe, un «palimpseste

19. Parmi de très nombreux exemples : Archivio di Stato di Napoli (désormais ASN), Archivio Borbone, 647, II, rapport sur Isaja De Vicariis, Naples, 1830, évoquant la très forte diffusion de médailles sectaires à l'effigie des martyrs parmi les sociétés secrètes politiques du Principat Ultérieur, autour d'Avellino.

20. Guglielmo PEPE, Naples selon l'histoire. Lettre à un baronet anglais sur l'Autriche en Italie, Paris, Fontemoing, 1840, p. 8 (sur la perspective générale) et 10 (sur Mario Pagano).

21. Guglielmo PePE, Memorie, Paris, Baudry, 1847, vol. 1, p. 36. Issu de la haute noblesse des Pouilles, fondateur de la République parthénopéenne en 1799, Ettore Carafa a été guillotiné le 4 septembre 1799. 
révolutionnaire $»^{22}$. Ils sont en revanche très peu nombreux à être nommés, à l'exception des grands patriotes de 1799 : le martyre politique constitue alors, pour les libéraux, une réalité collective, celle de la nation napolitaine, envisagée comme partie intégrante d'une mémoire libérale à plus large échelle, occidentale ou italienne.

Les écrits consacrés aux martyrs connaissent un développement considérable dans les années 1840, révélant la sensibilité accrue de la société méridionale à la mort politique ${ }^{23}$. Ils sont liés à une importante rhétorique libérale de la déploration, emblématique d'une lecture romantique de la nation comme «mélodrame» dont l'historienne Carlotta Sorba a fait l'un des traits communs aux discours patriotiques du Risorgimento ${ }^{24}$. Les publications relatives aux martyrs politiques se développent dès 1846, d'abord introduites par colportage ${ }^{25}$, puis publiées de façon légale sous le gouvernement révolutionnaire de $1848^{26}$. Cette évolution, qui suit la chronologie politique du royaume méridional, en lien avec la législation sur la presse, apparaît inverse à celle observée pour la France par Emmanuel Fureix, où la pratique du martyre politique tend à s'estomper dès le début des années 1840, alors qu'elle avait jusque-là une importance sociale nette, appuyée sur des nécrologes nombreux et largement diffusés ${ }^{27}$. À Naples, le tournant des années 1840 est caractérisé par la reconnaissance nominative des martyrs, considérés non plus comme les figures célèbres d'une histoire nationale continue et linéaire, mais comme des individus dont la vie et la mémoire sont liées à un contexte local. De ce point de vue, le cas du jeune libéral Luigi La Vista (1826-1848) apparaît emblématique. Élève de l'écrivain et critique Francesco De Sanctis, camarade et ami de Pasquale Villari et de Luigi Settembrini, il a été formé dans les milieux libéraux de la capitale alors en profond renouvellement ${ }^{28}$. Cet historien et philosophe avait commencé à écrire, le 11 mai 1848, « une série de biographies de nos martyrs politiques », à partir de ceux de 1799, à des fins de

22. Alain Hugon, Naples insurgée, 1647-1648. De l'événement à la mémoire, Rennes, Presses Universitaires de Rennes, 2011, p. 21.

23. Diego CARNEVALE, L'affare dei morti: mercato funerario, politica e gestione della sepoltura a Napoli (secoli XVII-XIX), Rome, École française de Rome, 2015.

24. Carlotta SORBA, Il melodramma della nazione. Politica e sentimenti nell'età del Risorgimento, Rome-Bari, Laterza, 2015.

25. C'est le cas de l'opuscule de Giuseppe MAZZINI, Ricordi dei fratelli Bandiera e dei loro compagni di martirio in Cosenza il 25 luglio 1844 : documentati colla loro corrispondenza, Bastia, Fabiani, 1846.

26. Parmi les nombreux textes publiés dans le royaume : Biagio MiRAGLIA, I martiri di Cosenza, Naples, s.n., 1848 ; Padre Serafino da Siracusa, Orazione funebre per gli estinti martiri della rigenerazione napolitana del 15 maggio 1848, Syracuse, Pulejo, 1848.

27. Emmanuel Fureix relève la transition vers de nouvelles pratiques de mobilisation politique comme les pétitions réformistes et les banquets politiques. Voir La France des larmes..., op. cit., p. 465 et suivantes.

28. Voir, sur le contexte intellectuel, Ennio Corvaglia, Le due Italie. Giovanni Manna e l'unificazione liberale, Naples, Guida, 2012, chap. 1. Quelques mots sur Luigi La Vista dans R. DE LORENZO, « La tradizione del 1799 nel Risorgimento italiano », art. cit., p. 357-358. 
« contemplation $»^{29}$. Le texte, que l'auteur n'a pas eu le temps de publier, mort le 15 mai 1848 dans les massacres perpétrés par l'armée bourbonienne, est resté à l'état de simple préface, mais il constitue le premier écrit napolitain entièrement consacré aux martyrs politiques de la patrie. Il en donne la première définition de son époque, évoquant des «saints de premier ordre », défenseurs acharnés de la liberté de la patrie, constituant un objet moral destiné à être admiré par la «patrie » et même dans le «monde ». Néanmoins, la liste des personnages qu'il incluait à cette catégorie ne nous est pas parvenue. On dispose en revanche de ses Note sulla rivoluzione delle Calabrie, à propos desquelles Pasquale Villari précise qu'elles constituent des «notules » destinées à fournir la matière à une histoire plus large des martyrs accordant une importance particulière au cas calabrais ${ }^{30}$. Ces Note, en partie fondées sur les mémoires qu'une de ses connaissances, le libéral de Reggio Saverio Vollaro (1827-1904) avait rédigées en prison $^{31}$, développent la répression des émeutes de Reggio en septembre 1847, conclues par le massacre de plusieurs jeunes patriotes à Gerace (2 octobre 1847). Vollaro lui semble constituer le parangon du martyr patriote, dans une période marquée par la très forte continuité du personnel politique. Né dans une famille de libéraux qui ont vécu les deux révolutions de 1799 et de 1820-21 réprimées par le roi bourbon Ferdinand IV, il raconte « les misères et cruautés des vingt-six dernières années », principalement la prison et la violence policière, et évoque les gloires militaires locales, comme les frères Plutino de Reggio ou les Romeo de San Stefano. À la suite d'autres libéraux contemporains, La Vista distingue en effet des traditions locales du martyre politique dont la Calabre apparaît l'exemple le plus significatif, à travers les cas des démocrates massacrés à Cosenza (1844) et des jeunes libéraux fusillés à Gerace (1847).

\section{COSENZA (1844) ET GERACE (1847) : LA FABRIQUE LOCALE DES MARTYRS}

Les figures de martyrs calabrais ont très tôt revêtu un pouvoir de mobilisation important dans une région périphérique marquée depuis longtemps par la conflictualité sociale au niveau local, où le maintien de l'ordre est rendu difficile à la fois par la topographie et par la faiblesse du réseau routier et télégraphique. La place des éléments calabrais dans le monde

29. «Ultimo scritto di Luigi La Vista », in Memorie e scritti di Luigi La Vista, raccolti e pubblicati da Pasquale Villari, Florence, Le Monnier, 1863, p. 196-199.

30. « Note sulla rivoluzione delle Calabrie », in Ibid., pp.188-192.

31. Archivio di Stato di Reggio Calabria (désormais ASRC), Archivio Visalli, 1, f. 11. 
libéral méridional est notable depuis 1799 au point de susciter l'inquiétude des pouvoirs publics qui en font les principaux responsables de la révolution de 1820-21 et s'attachent à contrôler la mobilité des hommes entre Naples et les Calabres, en particulier les étudiants, jugés potentiellement séditieux ${ }^{32}$. La situation littorale de la Calabre entre les mers Tyrrhénienne et Ionienne (carte 1), la proximité de la Sicile ont facilité le développement d'un mouvement démocratique italophile appuyé sur de forts liens avec Corfou où affluaient des exilés italiens en grande partie acquis aux idées mazziniennes ${ }^{33}$.

32. Sur la circulation des hommes entre les Calabres et Naples : Laura Di FIORE, Alla frontiera. Confini e documenti di identità nel Mezzogiorno continentale preunitario, Soveria Mannelli, Rubbettino, 2013. Sur les étudiants, on trouvera des indications générales dans Giuseppe PALMISCIANO, «Gli studenti universitari nell'Ottocento borbonico : fonti e indirizzi di ricerca », Rassegna storica salernitana, 35, 1999, p. 143-162.

33. Voir par exemple, sur le mouvement libéral-démocrate calabrais, Domenico Di GIORGIO, Benedetto Musolino e il Risorgimento in Calabria, Reggio, Historica, 1953. 


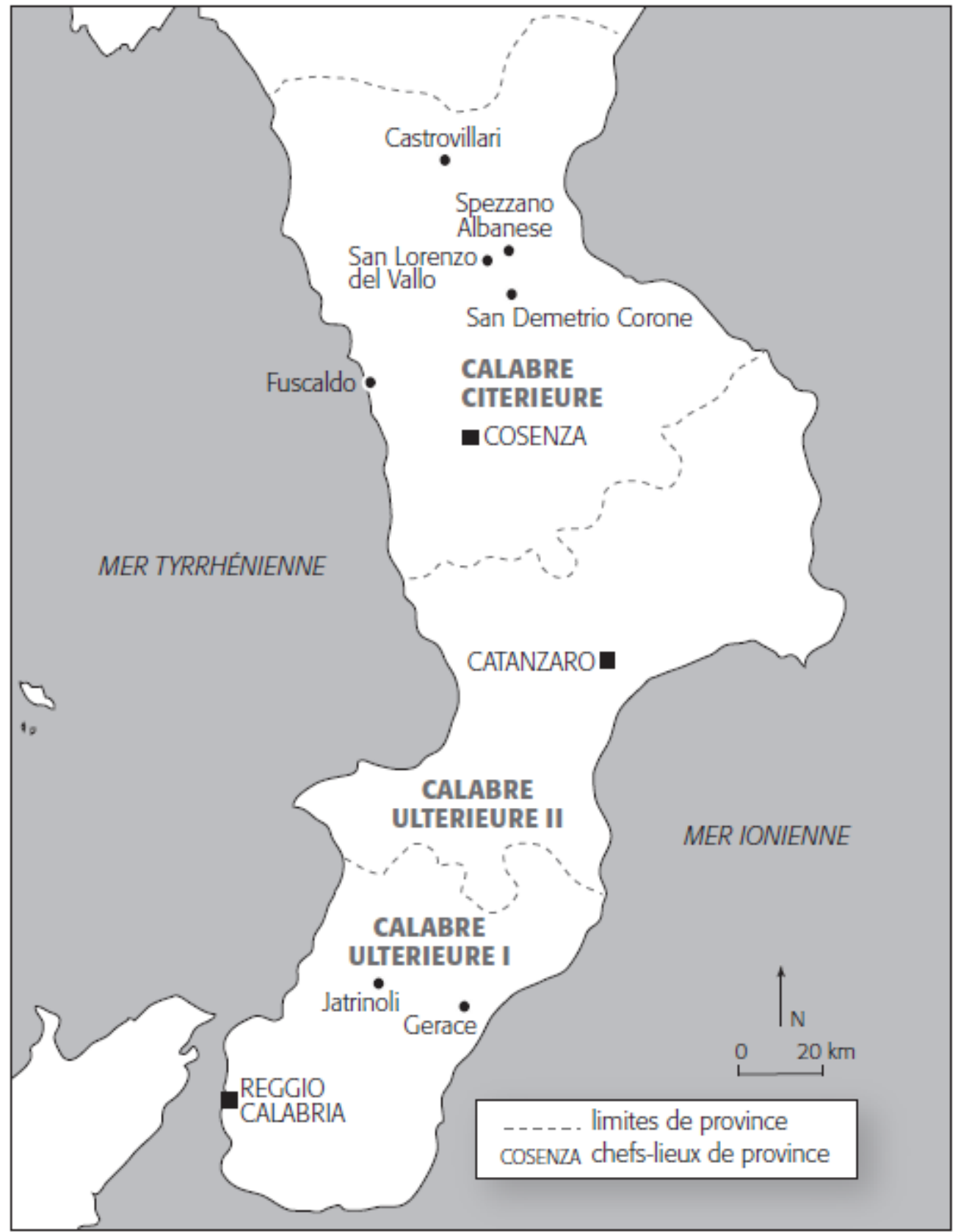

Carte 1. Les provinces de Calabre en 1848

(d'après Biagio Marzolla, Atlante corografico, storico e statistico del Regno delle Due Sicilie eseguito litograficamente, compilato, e dedicato a S.M. il Re Ferdinando, Naples, tip. Militare, 1832).

Un tel mouvement, unique dans un royaume où l'opposition modernisatrice est traditionnellement libérale-modérée, s'inscrit dans un champ politique complexe et hybride, unissant modérés et démocrates contre les Bourbons, le patriotisme primant sur les divergences idéologiques habituelles. Dans une chronique publiée après l'Unité italienne, un 
modéré de Cosenza, Davide Andreotti, les définit comme étant tous des «libéraux ${ }^{34}$. La référence commune aux martyrs locaux a largement contribué à consolider ce mouvement. L'écho des martyrs de 1844 a été immédiat chez certains exilés, y compris extérieurs à la Calabre $^{35}$. Dans le royaume, les publications se multiplient une fois la révolution de 1848 déclenchée, établissant des relations de parenté entre les martyrs de 1844 et de 1847, considérés comme les héros d'une cause patriotique commune dont ils seraient, par leur héroïsme naturel rapporté à leur identité calabraise, les meilleurs défenseurs. Un auteur comme Biagio Miraglia (1814-1885), médecin psychiatre originaire de la province de Cosenza, érige en héros les «martyrs de Cosenza » de 1844 dont ceux de Gerace seraient les héritiers directs, dans une série de quatre chants versifiés diffusés à Naples sous forme de tracts dans les premiers mois de $1848^{36}$. Miraglia, qui a quitté la Calabre en 1831 pour étudier puis exercer la médecine dans la capitale, définit la culture du martyre comme l'un des traits fondamentaux du caractère calabrais, décrit comme héroïque, à un moment où la littérature ethnographique et identitaire observe des « caractères nationaux » fondés sur des vertus et des vices propres à un espace donné ${ }^{37}$, et où les observateurs étrangers, qu'il s'agisse de voyageurs ou de diplomates, développent la violence et l'insoumission chronique des Méridionaux $^{38}$. Les libéraux calabrais n'y font pas exception, ayant consacré dans les années 1840 de larges passages de leurs journaux savants locaux, le Calabrese à Cosenza et la Fata Morgana à Reggio, à la description des us et coutumes propres à leurs provinces. Néanmoins et à la différence des évocations précédentes, Miraglia s'efforce de citer nommément les patriotes morts à Cosenza, rappelant leur origine sociale, principalement populaire (22 des 47 condamnés à mort étaient des paysans) et la variété des sentences (la mort, la condamnation aux fers et, dans une moindre mesure, la prison) ${ }^{39}$. Son analyse illustre donc l'importance du facteur local dans la mémoire politique des martyrs qui a constitué un des ressorts majeurs de la politisation révolutionnaire des trois Calabres. Les martyrs apparaissent ainsi les initiateurs

34. Davide ANDREOTTI, Storia dei Cosentini, Naples, Salvatore Marchese, 1874, vol. III, p. 295.

35. C'est le cas du Napolitain Giuseppe Ricciardi (1808-1882), auteur d'une brochure consacrée aux martyrs de Cosenza en circulation parmi la communauté italienne de Paris: Alla santa memoria di Attilio ed Emilio Bandiera, Domenico Moro, Niccolò Ricciotti, Anacarsi Nardi, Francesco Berti, Jacopo Venerucci, Jacopo Rocca e Domenico Lupatelli, morti per la libertà italiana in Cosenza a'25 luglio del 1844, Paris, Lacombe, 1844.

36. I martiri di Cosenza, di B.G. Miraglia, Cosentino (1844), Aversa, s.n., 1864, troisième édition sur la seconde de 1848.

37. Silvana PATRIARCA, Italian Vices. Nation and Character from the Risorgimento to the Republic, CambridgeNew York, Cambridge University Press, 2010.

38. Rosa Maria Delli QUADRI, Nel Sud romantico. Diplomatici e viaggiatori inglesi alla scoperta del Mezzogiorno borbonico, Naples, Guida, 2012.

39. La liste, citée dans le chant III des Martiri, est détaillée dans le Giornale del Regno delle Due Sicilie, 159, 29 juillet 1844, p. 157. 
d'un processus révolutionnaire beaucoup plus large, diffusé en 1848 dans toute la péninsule italienne et auprès des révolutionnaires européens ${ }^{40}$. De telles analyses contrastent avec celle avancée par les partisans de l'État bourbon, dont la presse officielle criminalise les révoltes calabraises, réduites à des «orgies» orchestrées par des «brigands » qui cherchent «davantage le vol que la politique », légitimant ainsi la répression comme une simple opération de maintien de l'ordre, apte à provoquer « la satisfaction de tous $»^{41}$.

À Reggio et à Cosenza, la mémoire des martyrs a été diffusée par une très large production de tracts, permise par la libéralisation de l'expression publique. Ces textes, qui développent principalement la violence exercée par l'État bourbon, rappellent les moments fondateurs de la révolte ${ }^{42}$. Ils mettent en scène le deuil des compatriotes sacrifiés, cités par leurs noms, considérés comme représentatifs du « génie calabrais », rappelant que tous ont été massacrés par l'armée ${ }^{43}$. Ils entendent par là rendre aux martyrs le culte qui leur est dû : en 1848, Miraglia invite les patriotes du royaume à commémorer les martyrs de Cosenza, devenus l'emblème d'une ville « belle comme une tombe peut être belle ${ }^{44}$. Le journal dont il est directeur, le Calabrese rigenerato, ouvre sa première édition du 15 février 1848 par une souscription pour faire ériger un mausolée aux martyrs de 1844 afin d'en faire les pères fondateurs de la révolution de 1848, alors à l'œuvre depuis quelques semaines ${ }^{45}$. Le monument de marbre, illustré par Giovanni Battista Santoro (1809-1855), un jeune peintre de Cosenza proche du courant libéral modéré et spécialisé dans la peinture sainte ${ }^{46}$, est finalement édifié en mars 1848 dans la nef de la cathédrale, et mentionne les noms de tous les martyrs de 1844. On perçoit, à travers la mise en scène votive des martyrs de 1844, l'instrumentalisation religieuse de la mémoire. En mars 1848, le député-maire de Cosenza, Tommaso Ortale (1802-1854), un avocat proche du courant démocrate, propose d'organiser des obsèques officielles le jour-même de l'inauguration du mausolée, «avec toute la pompe possible », pour rendre grâce à des hommes dont il avait fait cacher les dépouilles dans une

\section{Ibidem, p. 159.}

41. Giornale del Regno delle Due Sicilie, supplément au n ${ }^{\circ} 192$ du 4 septembre 1847, et 193, 6 septembre 1847. 42. Les textes rappellent l'exécution des martyrs de Cosenza le 25 juillet 1844, l'éclatement de l'insurrection de Reggio le 2 septembre 1847 et le massacre de Gerace le 2 octobre de la même année. Voir ASRC, Archivio Visalli, 1, f. 11, Pei 5 caduti sulla piana di Gerace nel 2 ottobre 1847, Reggio, s.d., Risposta ad un'articolo del giornale La Costituzione, Gerace Marina, marzo 1848 ; Il Quindici Settembre 1847. Sonetti, Reggio, s.n., 1848 ; Gli ultimi fatti di Calabria, s.l., s.d.

43. Biagio MiRAgLiA, Il genio Calabrese sui fatti di Reggio, Reggio, décembre 1847; Alla memoria degl'intrepidi giovani Bello, Ruffo, Mazzone, Verducci, Salvatore, fucilati in Gerace (1847), Reggio, 1848.

44. I martiri di Cosenza, op. cit., chant III, « Le prigioni di Cosenza », p. 31.

45. Il Calabrese rigenerato, $\mathrm{n}^{\circ} 1,15$ febb. 1848, particulièrement Alessandro CONFLENTI, « Ai lettori », p. 1, et Biagio MiRAGLIA, «Cronica », p. 2-3.

46. Carlo MASSINISSA PRESTERA, «La rigenerazione italiana », Il Calabrese rigenerato, $\mathrm{n}^{\circ} 2,29$ février 1848, $\mathrm{p}$. 9. 
chapelle de la commune ${ }^{47}$. La cérémonie, qui a lieu les 14 et 15 mars pour le quatrième anniversaire de la révolte de 1844, met à jour des cortèges funèbres, escortés par le clergé local, par des confréries laïques et par l'armée, et célèbre le «premier cri de la liberté » en même temps qu'il exprime le deuil collectif et le triomphe de la révolution. Le mausolée luimême, abondamment décrit dans le Calabrese rigenerato, associe, de manière classique pour l'Italie de l'époque, la symbolique patriotique et libérale à celle de la religion, flanqué de quatre statues féminines célébrant l'Italie, la liberté, la victoire et la force, surmontées de celle de la religion ${ }^{48}$. Le lien entre politisation libérale et religion, déjà visible dans la révolution de 1820-21 à travers l'importance de l'engagement révolutionnaire du bas clergé ${ }^{49}$, s'explique en très grande partie par le succès du néoguelfisme dans le royaume méridional et par la popularité du pape Pie $\mathrm{IX}^{50}$, qui s'est imposé comme l'un des protagonistes de la lutte nationale italienne depuis son avènement en 1846. Au culte du corps des martyrs s'ajoute celui du drapeau tricolore, considéré comme un «legs sacré » des martyrs de 1844 qui l'ont «baigné de leur sang » ${ }^{51}$. Défendre le drapeau tricolore revient donc à honorer une relique, et en ce sens, l'appel à la mobilisation pour l'Italie s'appuie sur les ressorts rhétoriques de la croisade. L'importante orchestration religieuse et festive des célébrations en témoigne, de même que son interprétation millénariste. Le frère Raffaele Orioli, un dominicain de Cosenza, prédit le jour des obsèques des martyrs que «quarante mille Calabrais » repousseront le «démon septentrional» (l'Autriche) pour permettre le règne du drapeau tricolore ${ }^{52}$. L'importante mobilisation des élites politiques et religieuses traduit l'importance du thème martyrologique dans la société cosentine de la fin des années 1840 : la mémoire des martyrs de 1844 semble être un instrument possible de la politisation des masses calabraises,

47. Ibidem, p. 8. Il s'agit de la chapelle Sant'Agostino, voisine du lieu d'exécution des martyrs.

48. Francesco Maria SCAGLIONE, «Esequie dei martiri di Cosenza », Il Calabrese rigenerato, $\mathrm{n}^{\circ} 4,2$ avril 1848, p. $25-26$.

49. Pour la province de Cosenza, quelques indications générales dans Gustavo VALENTE, «Il clero di Calabria Citra nel Risorgimento », Rassegna storica del Risorgimento, 40, 1956, p. 576-581.

50. La réception méridionale de la pensée de Vincenzo Gioberti et, plus largement, du néoguelfisme sont mal connus. Exposée dans le Primato morale e civile degli Italiani (1843), cette théorie consiste à fonder une confédération d'États italiens sous l'autorité du pape. On pourra se référer à l'article ancien de Teresa GIUFFRE, «La fortuna del giobertismo nell'Italia meridionale », Archivio storico per le provincie napoletane, 66, 1941, p. 99-148 et 68,1943 , p. $151-181$.

51. Il Calabrese rigenerato, ${ }^{\circ} 1,15$ février 1844, p. 6.

52. Ibidem, p. 8. Sur la symbolique révolutionnaire de 1848 en Calabre, voir Antonio ButTigLIONE, Il tricolore, il tamburo, la croce. La rivoluzione del 1848 in una provincia del Regno delle Due Sicilie, mémoire de tesi di laurea, Università degli Studi della Calabria, 2013. 
faiblement acquises aux idées libérales et qui ont constitué un soutien des contrerévolutionnaires en $1799^{53}$.

Les commémorations se multiplient ensuite, reproduisant l'exemple des funérailles des martyrs de Cosenza. En mars 1848 à Jatrinoli, près de Reggio, le maire Giuseppe Ventre, de tendance démocrate, fait construire sur la place publique du village un mausolée en l'honneur du martyr Domenico Tutini et invite, par l'intermédiaire de l'un des auteurs du Calabrese, l'écrivain Alessandro Conflenti, les villages voisins à suivre l'initiative ${ }^{54}$. De la même manière, des spectacles de théâtre joués à Cosenza et à Reggio commémorent la geste des martyrs de Gerace. Ils soulignant leur rôle dans le déclenchement de la révolution de 1848 et dans l'indépendance italienne, rappelant leur parenté avec les révolutionnaires de 1799, les martyrs chrétiens et surtout avec le pape Pie IX, alors considéré depuis le début de son pontificat en 1846 comme l'un des soutiens principaux des révolutions politiques italiennes ${ }^{55}$.

Les protagonistes locaux de la révolution de 1848 s'efforcent donc de célébrer la mémoire des martyrs par des commémorations ritualisées qui en font des emblèmes du patriotisme, des héros de la cause italienne et des modèles de vertu chrétienne. Parallèlement à ces pratiques officielles se développent des initiatives plus spontanées, dans des proportions que les sources policières et judiciaires locales, souvent lacunaires et focalisées sur la loyauté ou non au roi, ne permettent pas d'évaluer précisément. Les autorités policières de Reggio notent ainsi quelques tentatives isolées de réhabilitation de la mémoire des martyrs de Gerace au cours de l'année 1848. Le cas le plus significatif est peut-être l'exhumation des cadavres des cinq martyrs par six habitants de Gerace dont quatre sont propriétaires, deux sont diacres et tous sont notifiés comme chefs révolutionnaires ${ }^{56}$. Alors qu'aucun n'a de lien familial avec les martyrs, ils exposent sur la place municipale les corps jusque-là enterrés dans la fosse commune et mettent en place une souscription locale pour financer des funérailles publiques. Le rituel répond au schéma classique du culte des martyrs révolutionnaires, ajoutant à la tradition de la mort héroïque l'exposition des reliques, garantie de la mémoire, jugée capable

53. Anna Maria RAO (éd.), Folle controrivoluzionarie. Le insorgenze popolari nell'Italia giacobina e napoleonica, Rome, Carocci, 2001.

54. Alessandro CONFLENTI, «Monumento eretto in Jatrinoli a Domenico Tutini », Il Calabrese Rigenerato, $\mathrm{n}^{\circ} 7$, 23 avril 1848, p. 40. Sur l'auteur, voir Leonardo FALBO, «Il roglianese Alessandro Conflenti tra storiografia risorgimentale e bibliografia », in Giuseppe MASI (éd.), Tra Calabria e Mezzogiorno. Studi storici in memoria di Tobia Cornacchioli, Cosenza, Pellegrini, 2007, p. 125-149.

55. Alessandro CONFLENTI, « Notizie del nostro teatro », Il Calabrese Rigenerato, $\mathrm{n}^{\circ} 2$, 29 février 1848, p. 14-15. 56. ASRC, Atti di Polizia, 2, fuori busta, 1848, liste des capi, secondatori et gregarii suspectés d'avoir participé aux émeutes de 1848. 
de mobiliser le peuple parce qu'elle rappelle la violence exercée par l'État bourbonien ${ }^{57}$. En ce sens, les rituels votifs ont une fonction expiatoire et soutiennent une large rhétorique de la vengeance par laquelle les chefs révolutionnaires appellent à combattre l'armée et à tuer le roi au nom des exactions dont ils sont responsables.

\section{VENGER LES MARTYRS, EXPIER LES MASSACRES}

Les brochures diffusées à Cosenza et surtout à Reggio dans les premiers mois de la révolution présentent donc les deux martyres comme des massacres perpétrés par l'État sur des patriotes et des chrétiens dont ils rappellent avec insistance la loyauté au roi. La commune de Gerace, par exemple, n'a connu que très peu de révoltes avant 1847 à l'exception de quelques actes ponctuels comme la rébellion d'un jeune étudiant, Michele Bello, qui a détruit plusieurs statues du couple royal en 1846. Mais l'acte est resté isolé et c'est plutôt dans les conditions économiques des paysans locaux et dans le poids de la fiscalité qu'il faut rechercher les moteurs de la révolte de septembre $1847^{58}$. Un tract publié à Gerace en février 1848 rappelle par exemple que la bourgade «a toujours été loyale au roi et, pour son attachement à la loi, massacrée », disculpant les libéraux des accusations outrancières de républicanisme portées par les autorités bourboniennes et plaçant par là-même le pouvoir bourbon hors-la-loi puisqu'en massacrant des civils, il a violé à la fois un serment politique et les lois de la guerre ${ }^{59}$. Ces réflexions sont reprises dans une série de poèmes destinés à glorifier l'une des figures locales du patriotisme, Domenico Romeo (1796-1847), tué le 15 septembre 1847 par les gardes urbaines de Reggio, appelant à venger la mémoire de ce martyr local pour la cause italienne et pour la constitution ${ }^{60}$. Tous ces textes, anonymes, développent la violence du régime bourbonien dont ils concluent à la barbarie, en même temps qu'ils rappellent la loyauté des libéraux calabrais à la monarchie constitutionnelle mise en place début février 1848. Le roi apparaît donc comme un traître qui s'expose à la «vengeance de Dieu ${ }^{61}$ que prennent en charge les sympathisants de la cause italienne, au nom du compromis néoguelfe unissant les patriotes au pape. On retrouve là encore la rhétorique de la

57. Sur ce modèle, voir Antoine de BAECQUE, Le corps de l'histoire. Métaphores et politique (1770-1800), Paris, Calmann-Lévy, 1993, ch. III, «L'offrande des martyrs : le corps meurtri de la révolution », p. 343-374.

58. Vincenzo CATALDo, Cospirazioni, economia e società sul distretto di Gerace e in provincia di Calabria Ultra prima dal 1847 all'Unità d'Italia, Ardore Marina, Arti Grafiche Edizioni, 2000.

59. ASRC, Visalli, 1, f. 11, Risposta ad un'articolo del Giornale La Costituzione, op. cit.

60. ASRC, Visalli, 1, f. 11, « La vendetta ».

61. ASRC, Visalli, 1, f. 11, I martiri di Cosenza. 
croisade, largement exploitée par le clergé local, séculier ou non, dont les discours fortement moralisés font de l'opposition au roi une guerre juste. À Cosenza, le prêtre Zicarelli ou le moine dominicain Orioli appellent par exemple les Calabrais à «gagner la couronne du martyre » en combattant pour la liberté et pour l'Italie, afin d'honorer le drapeau tricolore baigné du sang des martyrs de $1844^{62}$. Ces postures radicales se renforcent et tendent à se généraliser lorsque la monarchie constitutionnelle connaît ses premiers échecs, en avril 1848, imputés à la trahison du roi, incapable de respecter la monarchie constitutionnelle qu'il a accepté de mettre en place deux mois plus tôt. Contre un roi napolitain parjure, l'option italienne s'impose donc comme légitime, renforcée par des références fréquentes aux pères littéraires du Risorgimento, Dante et Machiavel, bien connus des élites locales cultivées. Les libéraux calabrais y recherchent des principes d'action politique qui permettraient de défendre la mémoire des martyrs. De ce point de vue, la fraternité italienne constitue une stratégie défensive pour venger les cinq fusillés de Gerace. Le poète Carlo Massinissa Presterà (18161891), bien que modéré, fait appel aux fratelli d'Italia, évoquant le chant indépendantiste du même nom écrit par deux jeunes mazziniens génois un an plus tôt :
«Deh! sorgete, o fratelli d'Italia
Vendicate i fratelli caduti
Son già fatti cadaveri muti
Quei che tanto di patria parlar
Son cadaveri ! il sangue ringurgita
Delle fragili zolle imbevute:
Fra quel sangue la vostra salute
Se saprete vendetta cercar ${ }^{63}$.

En reprenant la rhétorique fraternelle et insurrectionnelle du poème mazzinien, Massinissa Presterà appelle les patriotes italiens à un mouvement coordonné. L'identité calabraise, clairement revendiquée par les auteurs de ces tracts, devient donc une identité patriotique italophile, incarnée par les figures des martyrs. Venger les martyrs revient donc à construire l'Italie en leur nom. Les deux identités ne sont pas exclusives l'une de l'autre, mais elles sont

62. Les discours, qui datent du début de l'année 1848 , sont cités dans $I l$ Calabrese rigenerato, $\mathrm{n}^{\circ} 1,15$ février 1848, p. 8, et n ${ }^{\circ} 4,2$ avril 1848, p. 27.

63. ASRC, Visalli, 1, f. 11, Alla memoria degl'intrepidi giovani Bello, Ruffo, Mazzone, Verducci, Salvatore, fucilati in Gerace 1847, Cosenza, 1848. Traduction :

« Ah ! levez-vous, ô frères d'Italie,

Vengez vos frères morts au combat,

Ce sont déjà des cadavres muets

Ceux qui parlaient tant de la patrie

Sont des cadavres ! Leur sang régurgite

Des fragiles mottes de terre qui ne l'ont pas absorbé

Dans ce sang sera votre salut

Si vous savez chercher la vengeance !» 
imbriquées et complémentaires. Cette conversion à l'italianité, sans être générale, est fréquente dans les stratégies politiques des libéraux; néanmoins, si le roi est rendu responsable du sacrifice des martyrs calabrais, les patriotes qui appellent au régicide ou s'affichent ouvertement partisans de la république demeurent numériquement minoritaires. Sur 172 condamnés pour outrage au roi dans la province de Reggio en 1848, seuls 13 le sont pour des faits remontant aux premiers mois de 1848, parmi lesquels seuls 3 auraient manifesté en public des opinions républicaines ${ }^{64}$. Dans le cas de Cosenza, la chronique d'Andreotti confirme cette dissociation entre libéralisme, projet italien et république : il rappelle qu'en 1844, « l'idée d'unifier l'Italie sous une monarchie constitutionnelle était très diffusée dans la province $»^{65}$.

Les conditions et les modalités de la vengeance évoluent aux lendemains du massacre du 15 mai 1848 où les exactions sur des civils ont fait scandale et où les députés au Parlement napolitain font l'objet d'une héroïsation immédiate ${ }^{66}$. Guglielmo Pepe rappelle, avec une exagération certaine, que la nation napolitaine les a tout de suite considérés comme «les dignes fils et descendants de ces immortels martyrs », précisément ceux de la révolution de $1799^{67}$. Dans les Calabres, la nouvelle des massacres est immédiatement connue par le retour de plusieurs patriotes à forte influence locale comme l'ancien inspecteur des Postes Achille Parise ou encore Pietro Salfi, neveu du célèbre exilé Francesco Saverio Salfi (1759-1832) et correspondant régulier de la presse savante de Cosenza. Les réseaux épistolaires sont également déterminants: Biagio Miraglia, par exemple, correspond depuis Naples avec les principaux journaux calabrais et publie dans la capitale ses Martiri di Cosenza qui lui valent d'être mis en prison en $1849^{68}$. Rappeler le martyre des patriotes calabrais en soulignant leur héroïsme face à l'armée d'un roi parjure permet d'amplifier la mobilisation calabraise, qui se radicalise considérablement. La vengeance se réoriente dès lors contre le roi Ferdinand, jugé responsable d'une série de massacres qui ont contribué à enrichir le martyrologe patriotique. Un patriote calabrais, Francesco De Rosa, dont le chroniqueur Davide Andreotti rappelle qu'il est l'un des rares républicains assumés ${ }^{69}$, évoque une semaine après les massacres du 15 mai

64. ASRC, Atti di Polizia, 2, fuori busta 1848, cit.

65. D. ANDREOTTI, Storia dei Cosentini..., op. cit., vol. III, p. 295.

66. Sur le tournant du 15 mai 1848 dans l'évolution politique et sociale du royaume des Deux-Siciles, voir Viviana MELlone, «Dopo i fatti napoletani del 15 maggio 1848. Vicende giudiziarie ed indagini di alta polizia a confronto », Rivista Storica Italiana, 125, 2013/2, p. 497-550.

67. Guglielmo PEPE, Casi d'Italia negli anni 1847, 48 e 49: continuazione delle memorie del generale Guglielmo Pepe, Gênes, s.n., 1851.

68. B. MiRAGLIA, I martiri di Cosenza ..., op. cit.

69. D. ANDREOTTI, Storia dei Cosentini..., op. cit., vol. III, p. 312. 
les «frères napolitains mitraillés par le tyran destructeur (bombardatore) » ${ }^{70}$. Au même moment, dans une proclamation diffusée à Cosenza avec les démocrates Raffaele Valentini, Domenico Mauro et Eugenio De Riso, Giuseppe Ricciardi, venu de Naples pour soutenir la mobilisation calabraise, évoque l'impossible consensus autour d'un roi-traître qu'il faut désormais combattre ${ }^{71}$. Le recours à partir du mois de juin à un nouvel organe de presse beaucoup plus radical, édité par le Comité de Salut public de Cosenza, L'Italiano delle Calabrie, atteste cette évolution, s'ouvrant sur une proclamation à tous les Calabrais donnant à voir un tableau tragique des massacres du 15 mai dont il incombe aux patriotes de venger les victimes. Les auteurs décrivent la cruauté des gardes suisses qui, sous les ordres du roi, auraient profané des lieux de culte, arraché des enfants des bras de leurs mères et les auraient étranglés sous leurs yeux ${ }^{72}$. Un tel tableau, largement outrancier, a pour fonction de mobiliser les masses contre la royauté : à partir de là, les auteurs exhortent le peuple à prendre les armes, invoquant le souvenir de la victoire des Calabrais sur l'armée française d'occupation en 1806, encore largement présent et entretenu par la presse locale qui lui a consacré plusieurs $\operatorname{articles}^{73}$.

Ce mouvement de radicalisation se lit également à travers les pratiques politiques populaires, marquées par l'accroissement et la systématisation de la violence, physique ou symbolique, contre le roi, ses symboles ou ses représentants, justifiée par l'expiation des massacres. Les actes de violence sur la police et l'armée demeurent marginaux. En revanche, les tracts et les journaux diffusés dans la province et lus dans des lieux classiques des sociabilités révolutionnaires (cafés, maisons privées, magasins où sont organisées des réunions politiques) contribuent à diffuser l'appel à la rébellion. Dans les villages, les prêtres en sont des relais privilégiés : les prêches régicides sont fréquents, dans une région où la majorité du clergé est libérale ou démocrate. À Castrovillari, au nord de la Calabre Citérieure, le père Michele Bellizzi, prêche le 3 juin 1848, lors des funérailles des martyrs du 15 mai, contre un roi-tyran qui mérite la mort et dont le sang pourrait servir d'engrais à d'éventuels

70. Lettre de Francesco De Rosa à Nicola Palladini, Cosenza, 24 mai 1848, citée in Documenti storici riguardanti l'insurrezione calabra, preceduti dalla storia degli avvenimenti di Napoli del 15 maggio, Naples, Araldo, 1849, p.118.

71. Proclamation de Raffaele Valentini, Giuseppe Ricciardi, Domenico Mauro, Eugenio De Riso, Cosenza, 2 juin 1848, citée in Ibidem, p.127-128: «Les énormes faits de Naples du 15 mai, et les actes qui ont détruit la Constitution, qui l'ont mise en retrait, ont rompu toute forme de pacte entre le Prince et le Peuple ».

72. « Ai generosi abitanti delle Calabrie », L'Italiano delle Calabrie, $\mathrm{n}^{\circ} 1,7$ juin 1848, p. 1-2.

73. Ibid. : «Et la vengeance sera faite. Rappelez-vous, frères, les vingt mille Français, commandés par Masséna, qui trouvèrent ici leur tombe !». Sur la campagne de Calabre de 1806-1807, voir N. CADET, Honneur et violences de guerre..., op.cit. 
arbres de la liberté ${ }^{74}$. Le cas n'est pas isolé : l'insulte régicide est l'une des formes les plus courantes de cette violence expiatoire. C'est essentiellement par des pratiques iconoclastes qu'on cherche à venger la mémoire des martyrs, principalement dans les villages ruraux et périphériques de la province de Cosenza, pour lesquelles sont condamnés 564 sujets pour l'année 1848, essentiellement en juin et juillet ${ }^{75}$. De telles pratiques impliquent une violence ritualisée, orientée contre les symboles et emblèmes royaux, phénomène dont la place dans les révolutions de l'époque contemporaine est en cours de réévaluation ${ }^{76}$. Les statues, les armes de la royauté, les drapeaux présents dans les édifices publics sont exposés sur la place du village, avant d'être détruits en public par des coups de fusil, de marteau ou de poignard. Le rituel, généralement collectif, s'accompagne de chants et d'insultes régicides et profite largement de l'appui des autorités civiles ou ecclésiastiques locales qui y sont souvent impliquées. Dans le village albanais de San Demetrio Corone au nord-est de Cosenza, le prêtre catholique Francesco Maria Lopez, proche des frères Mauro dont il fréquente les réunions politiques, s'empare de la statue du roi située dans la mairie, la recouvre de vêtements ridicules en l'insultant, puis la fracasse à coups de marteau. La destruction des effigies royales illustre donc la réciprocité de la violence politique qui entend expier le martyre par le sacrilège, en attentant au corps du roi. Un habitant de Fuscaldo au nord de Paola, Francesco Maria Lanzellotti, brise la statue du roi, place sa tête à l'extrémité d'un bâton et parcourt les rues du village en appelant à la rébellion contre le « roi maquereau (re marianaccio) ». D'autres substituent à l'effigie du roi déchu des images alternatives de la royauté : le portrait de Charles-Albert de Piémont-Sardaigne est affiché en lieu et place de celui de Ferdinand à San Lorenzo del Vallo près de Spezzano Albanese, ou à San Lorenzo, village de montagne de 1 'Aspromonte près de Reggio ${ }^{77}$. Près de la ville littorale de Paola, en Calabre Citérieure, on remplace le portrait du roi par ceux de Domenico Mauro ou d'autres radicaux comme Giuseppe Masciari ou Carlo Mileti, chefs révolutionnaires locaux considérés comme les dépositaires légitimes de la couronne ${ }^{78}$. La fonction de l'iconoclasme est alors l'imposition symbolique d'une république, qui rejoint la mobilisation armée contre la royauté dans les camps de révoltés de Paola et de Spezzano Albanese, aux entrées maritime et

74. ASN, Borbone, 1044, 35, Liste des condamnés politiques de la province de Cosenza.

75. Archivio di Stato di Cosenza (par la suite ASC), Processi politici, plusieurs dossiers.

76. Notamment Emmanuel FUREIX, «L'iconoclasme : une pratique politique (1814-1848) ? », in Laurent LE Gall, Michel OfFerle, François PlouX (éd.), La politique sans en avoir l'air. Aspects de la politique informelle XIX ${ }^{e}-X X I^{e}$ siècles, Rennes, Presses Universitaires de Rennes, 2013, p. 117-131; voir également Emmanuel FUREIX (éd.), Iconoclasme et révolutions (XVIII ${ }^{e}$-XX $X^{e}$ siècles), Seyssel, Champ Vallon, 2014.

77. Sur le dernier cas : ASRC, Atti di Polizia, 2, fuori busta, 1848, cité.

78. ASN, Borbone, 1044, 35, cité. 
terrestre de la province, pour défendre Cosenza contre l'arrivée des troupes royales du général Nunziante. Les sources policières montrent que pour beaucoup d'hommes, la destruction des statues royales a été un préalable à l'enrôlement dans les camps, montrant à la communauté villageoise un exemple d'héroïsme. En juin 1848, la levée d'une armée informelle de volontaires achève le cycle de vengeance par le combat contre l'armée royale au nom de la santa causa des martyrs locaux et de leurs frères napolitains tombés le 15 mai ${ }^{79}$. La volonté d'expier les massacres et le parjure royal réactive donc des réflexes pré-politiques comme le tyrannicide ou le sacrilège : à l'exception des leaders révolutionnaires comme Domenico Mauro, le sens de la «république » que certains entendent mettre en place n'est pas connu, parfois confondu avec l'imposition d'un nouveau roi issu du peuple, et c'est souvent la rumeur de nouveaux massacres ou de la mort du roi qui dicte ces régicides symboliques ${ }^{80}$. Après l'échec de la révolution de 1848, la mémoire des martyrs calabrais est largement récupérée par les libéraux modérés en exil à Turin, qui l'incluent au martyrologe national de l'indépendance italienne.

\section{DES MARTYRS POUR L'ITALIE? LOCALISME ET NATIONALISME (ANNEES 1850)}

Les histoires immédiates qu'écrivent les libéraux en exil ont vocation à justifier les révolutions passées et à les inscrire dans la perspective d'un mouvement national italien qui se serait formé à la faveur d'expériences communes, notamment lors des campagnes militaires menées en Lombardie et en Vénétie contre l'occupant autrichien. En rappelant leur rôle fondateur dans les révolutions italiennes et leur opposition constante à un régime tyrannique, ils font des martyrs méridionaux des acteurs à part entière du Risorgimento, montrant la persistance de l'identité napolitaine à côté du patriotisme italien péninsulaire. Cette conversion des martyrs à l'italianité s'inscrit dans un mouvement plus large, souligné par l'historiographie, selon lequel les révolutions de 1848 auraient vu s'accélérer les projets de

79. Bollettino del Comitato di Salute Pubblica di Cosenza, ${ }^{\circ} 2,4$ juin 1848, repris dans Documenti storici riguardanti l'insurrezione calabra..., op. cit., p. 130.

80. Les exemples sont très nombreux dans la province de Cosenza pour les mois de juin et juillet 1848 , comme à Cassano (ASC, Processi politici, b. 32 et 37), à Riggiano (ASC, Processi politici, b. 35 bis), à Aprigliano (b. 88, f. 508) ou à Montalto (b. 88bis). Les pratiques du tyrannicide sont surtout connues pour l'époque moderne : voir Monique COTTRET, Tuer le tyran? Le tyrannicide dans l'Europe moderne, Paris, Fayard, 2009, ou encore Mario TURCHETTI, Tyrannie et tyrannicide de l'Antiquité à nos jours, Paris, PUF, 2001. 
construction nationale ${ }^{81}$. Guglielmo Pepe rappelle ainsi que les développements italiens de 1848 ont commencé en Calabre avec les émeutes de Reggio en septembre 1847 : en ce sens, des martyrs comme Domenico Romeo ou comme les fusillés de Gerace auraient eu l'initiative d'une expérience fondatrice de l'italianité ${ }^{82}$. Pour Giuseppe Massari (1821-1884), un autre patriote en exil à Turin après avoir été député pendant la révolution, les martyrs de 1848 sont à la fois héritiers des générations successives de patriotes napolitains et les protagonistes de la résistance héroïque des populations méridionales à la tyrannie des Bourbons ${ }^{83}$. Par-delà les distinctions classiques entre modérés et démocrates, il faut voir dans cette communauté de résistance à la tyrannie le poids des réseaux du libéralisme méridional qui unissaient les patriotes calabrais et napolitains. Trois des martyrs de Gerace, Gaetano Ruffo, Michele Bello et Paolo Mazzone, étudiants à Naples au début des années 1840, bien que démocrates, étaient membres du Comitato liberale du modéré Carlo Poerio, publiaient des articles dans les journaux libéraux napolitains et fréquentaient les milieux patriotiques de la capitale ${ }^{84}$. De la même manière, les réseaux transnationaux dans lesquels s'insèrent les exilés de l'après-1848 ont contribué à la fortune européenne des martyrs méridionaux, constitués en icônes de la résistance au despotisme, corroborant les écrits d'observateurs étrangers comme les Britanniques W.E. Gladstone ou Charles Macfarlane ${ }^{85}$. Dès lors, les martyrs sont l'incarnation des «horreurs de Naples », devenues un argument essentiel du débat diplomatique et social européen dans les années $1850^{86}$. Rappeler le martyre des patriotes de 1848 justifie donc à la fois la violence politique et le primat napolitain dans les révolutions italiennes en faisant des Méridionaux les meilleurs défenseurs de l'italianité, comme le rappelle Massari : «En l'espèce, les Napolitains montrèrent par le sang que cette calomnie lancée par l'étranger était fausse, les Italiens ne se battent pas ${ }^{87}$. Cet aphorisme fait des Napolitains des Italiens exemplaires, à la fois parce qu'ils incarnent le caractère national indépendantiste propre à la

81. Voir essentiellement Axel KÖRNER (éd.), 1848, a European Revolution? International Ideas ans National Memories of 1848, New York, Palgrave Macmillan, 2000.

82. Casi d'Italia negli anni 1847, 48 e 49: continuazione delle memorie del generale Guglielmo Pepe, Turin, s.n. 1851 .

83. G. MASSARI, I casi di Napoli dal 29 gennaio 1848 in poi..., op. cit., p. 6-9.

84. Sur le Comitato liberale, voir ASRC, Archivio Plutino, 13, f. 765, 23, ou encore Paolo PELLICANO, Ricordi intorno al movimento politico di Reggio nell'anno 1847, Naples, Morano, 1879, p. 12.

85. William E. Gladstone, Two Letters to Lord Aberdeen on the State Prosecutions of the Neapolitan Government, Londres, John Murray, 1851 ; Charles MACFARLANE, A glance at revolutionized Italy : a visit to Messina, and a tour though the Kingdom of Naples, the Abruzzi, the Marches of Ancona, Rome, the States of the Church, Tuscany, Genoa, Piedmont \&c \&c in the summer of 1848, Londres, Smith \& Elder, 1849, 2 vol.

86. Sur le rôle des libéraux napolitains exilés dans la formulation de la «question méridionale », voir Marta Petrusewicz, Come il Meridione divenne una Questione. Rappresentazioni del Sud prima e dopo il Quarantotto, Soveria Mannelli, Rubbettino, 1998 ; sur le débat international, voir Nelson J. MoE, The View from Vesuvius. Italian Culture and the Southern Question, Berkeley, University of California Press, 2002.

87. G. MASSARI, I casi di Napoli dal 29 gennaio 1848 in poi..., op.cit., p. 165. 
péninsule et parce qu'ils sont les héritiers de la longue tradition des martyrs napolitains. On perçoit alors la double appartenance patriotique de ces martyrs, à la fois des combattants au service de la liberté napolitaine et de la nation italienne. Cette situation ne présente pas d'équivalent parmi les autres patriotes de la péninsule, et constitue l'héritage du particularisme méridional, marqué depuis l'époque moderne par des revendications identitaires propres autour de la nazione napoletana. Le patriotisme napolitain tel qu'il s'exprime dans les années 1850 semble l'héritier de ces constructions, qui définissent la nation comme une collectivité fondée sur des habitus communs, quelle qu'en soit l'échelle géographique $^{88}$. Dans ces conditions, les figures de martyrs incarnent à la fois la patrie locale, toujours jugée première, et la patrie plus large représentée par l'Italie.

Cette double appartenance explique que les martyrs méridionaux soient par la suite intégrés aux initiatives mémorielles de la communauté des nombreux exilés italiens installés en Piémont, particulièrement à Turin alors qualifiée de « Mecque des exilés ${ }^{89}$. L'initiative la plus déterminante est le fait d'un avocat napolitain de tendance démocrate, Gabriele D’Amato, fondateur en 1851 de la Società del Panteon dei Martiri della Libertà Italiana chargée d'éditer les biographies de 96 martyrs ou groupes de martyrs supposés avoir contribué, de manière directe ou indirecte, à l'indépendance italienne que beaucoup d'exilés cherchent à construire aux côtés de la monarchie piémontaise. La société regroupe une trentaine de contributeurs, majoritairement méridionaux, ayant tous participé à la campagne de défense de Venise en 1848, auxquels s'ajoutent des associés démarchés par D’Amato luimême dans la totalité du royaume de Piémont ${ }^{90}$. Elle publie ainsi les biographies d'abord sous la forme de fascicules individuels, illustrés de lithographies exaltant l'héroïsme des martyrs, puis en deux volumes édités en 1851 puis en 1852. Dans sa préface, D’Amato en impute le succès considérable à la relative liberté d'expression qu'offre la législation piémontaise et au soutien politique et financier du ministère de Massimo D'Azeglio ${ }^{91}$. L'ensemble du processus d'édition est assumé par les exilés, impliquant notamment le marquis Diego Soria de Crispano, vétéran napolitain de la guerre d'indépendance grecque à laquelle il a consacré une

88. A. MUSI, « La nazione napoletana prima della nazione italiana », art. cit.

89. Ester DE FORT, «Immigrazione politica e clima culturale a metà Ottocento nel Regno di Sardegna », in Luca Lo BASSO (éd.), Politica e cultura nel Risorgimento italiano. Genova nel 1857 e la fondazione della Società Ligure di Storia Patria. Atti del convegno, Gênes, Società Ligure di Storia Patria, 2008, p. 193-223. Sur les parcours géographiques et sociaux des exilés : Gian Biagio FURIOZZI, L'emigrazione politica in Piemonte nel decennio preunitario, Florence, Olschki, 1979.

90. Archivio di Stato di Torino (désormais AST), Sezioni Riunite, Emigrati, I, 22, D’Amato (Gabriele).

91. Panteon dei martiri della libertà italiana, Turin, Stabilimento tipografico italiano, 1852, vol. 1, préface, p. I. 
histoire $^{92}$, installé à Turin comme éditeur, qui emploie plusieurs exilés méridionaux. Ce sont encore deux exilés, Francesco Guarino et le prêtre Giuseppe Del Re, qui se chargent de diffuser les volumes, l'un par colportage et l'autre dans la librairie napolitaine qu'il a ouverte à Gênes en 1852 pour l'usage exclusif de ses compatriotes ${ }^{93}$. Le produit des ventes et des souscriptions est destiné à financer une industrie, l'Opificio Nazionale Ligure, supposée subvenir aux besoins des très nombreux exilés regroupés à Gênes, sans se limiter aux seuls Méridionaux. Organisée par un prêtre lombard lui-même réfugié en Piémont et proche des élites turinoises modérées, l'abbé Carlo Cameroni, l'initiative revendique un «but moral et philanthropique », celui de faire «l'éducation politique » de tous les Italiens par l'exemple des martyrs. Elle veut ainsi s'apparenter à une « protestation des vaincus » visant à expier des tyrannies dont les Bourbons de Naples ont constitué l'exemple le plus évident ${ }^{94}$. Malgré l'ambition communautaire de l'ouvrage, qui entend contribuer à former la communauté imaginée des Italiens, leur répartition géographique est inégale :

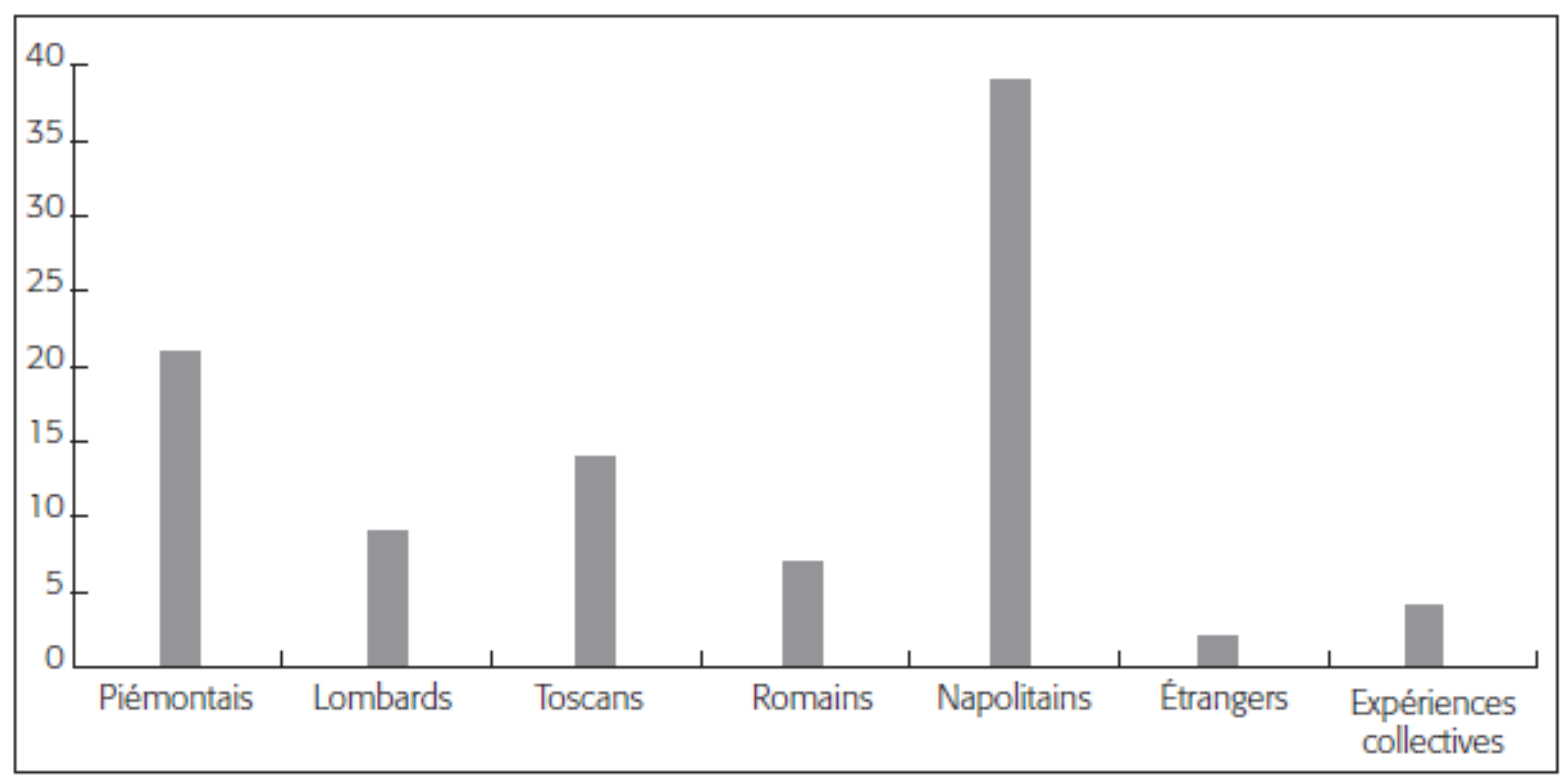

Graphique 1. Origine des 96 martyrs ou groupes de martyrs du Panteon dei Martiri della libertà italiana (1851-1852)

Les Méridionaux représentent la majorité des martyrs évoqués par l'ouvrage, avec $40 \%$ des notices. On y retrouve les patriotes de 1799, traités soit individuellement (Domenico

92. Diego SORIA, Istoria della Grecia dal 1824 in poi per servire da continuazione a quella del sig. Pouqueville, Naples, Diogene, 1840, 3 vol.

93. AST, Sezioni Riunite, Emigrati, I, 23, Del Re (Giuseppe).

94. Panteon dei martiri della libertà italiana..., op. cit., vol. 1, p. I-III. 
Cirillo, Ettore Carafa, Francesco Caraccciolo), soit dans des fiches collectives (« derniers défenseurs de la République parthénopéenne »), auxquels s'ajoutent les exilés de 1821 (Pietro Colletta) et les victimes des guerres de 1848 (Alessandro Poerio). À l'exception du Piémont dont certains des révolutionnaires des années 1820 sont commémorés (Santorre di Santarosa), tous les martyrs antérieurs à 1848 sont napolitains, rappelant encore une fois le caractère précurseur des Méridionaux dans le processus d'indépendance italienne. Les Napolitains sont d'autre part les seuls à disposer de notices collectives commémorant des martyrs populaires, acteurs des révoltes qu'a connues le royaume dans ses périphéries, dont toutes sont représentées. Le Calabrais Errico Poerio, cousin du député Carlo, alors en prison, et du poète Alessandro, mort au combat à Venise en 1848, rappelle ainsi l'exemple qu'ont constitué les martyrs de Cosenza ${ }^{95}$, alors que le Messinois Giacinto Scelsi inclut ceux de Gerace aux «martyrs de Messine » de 1847 auxquels il attribue l'origine de la révolution ${ }^{96}$. Il intègre ainsi les martyrs calabrais à une expérience plus large, celle du soulèvement des périphéries méridionales du royaume contre l'autorité des Bourbons. La Società del Panteon dei Martiri transpose donc dans la communauté des exilés de Turin la culture méridionale des martyrs, dont elle reprend à la fois la rhétorique expiatoire et le localisme. D'autres initiatives témoignent du rôle moteur des Napolitains : lorsqu'a lieu le quatrième anniversaire de la constitution piémontaise en 1853, un autre Méridional, Mariano D'Ayala, y voit l'occasion de commémorer les martyrs des campagnes militaires de 1848, publiant des listes révélant, une fois de plus, la prégnance des éléments napolitains ${ }^{97}$. Ces célébrations, effectuées dans la capitale piémontaise, bénéficient du soutien de la monarchie des Savoie et des élites turinoises, qui y trouvent un prétexte à leur projet d'inclusion du royaume des Deux-Siciles dans l'Italie en construction. Massimo d'Azeglio, premier ministre du royaume de 1849 à 1852, est personnellement lié aux Méridionaux réfugiés à Turin, par l'intermédiaire de l'abbé Cameroni qui organise le soutien aux réfugiés. Ce sont donc des réseaux humains qui ont facilité l'articulation entre les appartenances napolitaine et italienne, symbolisée par les figures de martyrs. Leur cas est néanmoins singulier : d'autres États italiens ont valorisé leurs martyrs locaux - la diversité géographique des cas traités par le Panteon l'illustre -, mais sans en faire les supports d'une identité locale jugée supérieure à celle de l'Italie unie. Les martyrs méridionaux servent donc à la défense et à la valorisation de la nation napolitaine, dont l'identité se maintient face au patriotisme italien, sans qu'il y ait contradiction avec celui-ci.

95. Errico POERIO, « Alcuni Martiri cosentini », Ibidem, t. I, p. 353-366.

96. Giacinto SCELSI, «I martiri di Messina (1847) », Ibidem, t. II, pp. 108-119.

97. Mariano D'AYALA, Non ti scordar di loro, Turin, Fontana, 1853. 
Cette fonction localiste, très présente dans le cas des Napolitains des années 1850, ne leur est cependant pas spécifique, puisqu'elle se retrouve dans d'autres espaces où le libéralisme s'est appuyé sur des lieux de mémoire locaux ou régionaux ${ }^{98}$.

Le royaume des Deux-Siciles a donc vu se former une culture libérale des martyrs, qui s'est affirmée dans les années 1840 et s'est nourri des victimes de la répression de 1848. Elle a constitué à la fois un puissant instrument de politisation locale, en particulier dans les Calabres, et l'un des ressorts majeurs de l'identité méridionale. Si une partie des libéraux en exil après 1848 essaie d'en faire des icônes du patriotisme italien, on voit persister les logiques communautaires méridionales, soulignant la singularité numérique et chronologique des martyrs napolitains et calabrais par rapport à ceux de la péninsule. Cette tendance se confirme alors que se forme le nouvel État italien en 1860 : des Napolitains comme Giuseppe Ricciardi ou Mariano D'Ayala cherchent dans leurs martyrs des pères fondateurs de l'Italie unie $^{99}$, alors que des Calabrais comme Carlo Massinissa Presterà ou Biagio Miraglia continuent à célébrer leurs compatriotes morts pour la liberté ${ }^{100}$. Après l'Unité également, on voit se développer les projets de monuments commémorant les martyrs révolutionnaires du Mezzogiorno, dont le plus célèbre est installé à Naples, piazza dei Martiri, en 1861. Ils témoignent de la récupération et de l'institutionnalisation de la mémoire des martyrs par le nouvel État italien. D’une culture de la résistance donc, la célébration des martyrs devient à la fois un support de la propagande italienne et de l'exception méridionale, valorisé par les élites napolitaines. On voit alors se complexifier et se généraliser l'usage des martyrs, au point que, pour Marc Monnier, italianiste genevois installé à Naples, l'Italie en cours d'unification est devenue « la terre des morts ${ }^{101}$, l'usage des figures héroïques des martyrs étant devenue un outil de politisation et de légitimation du patriotisme méridional. L’utilisation des ressorts du

98. L'historiographie en a montré plusieurs exemples dans des États centralisés dont certains espaces ont à la fois connu des particularismes identitaires et des formes locales de mobilisation politique, dont les exemples pourraient être multipliés. À Lyon en 1848, des constructions similaires sont relevées par Vincent ROBERT, Les chemins de la manifestation (1848-1914), Lyon, Presses Universitaires de Lyon, 1996, p. 88. À Barcelone à la fin du XIX ${ }^{\mathrm{e}}$ siècle, l'identité catalane a recouru à des figures de martyrs locaux : Stéphane MICHONNEAU, Barcelone. Mémoire et identité 1830-1930, Rennes, Presses Universitaires de Rennes, 2007, ch. 6, «Conflits de mémoire », p. 83-94.

99. Parmi d'autres textes : Giuseppe RICCIARDI, Martirologio italiano dal 1792 al 1860, Florence, Le Monnier, 1860 ; Mariano D’AYAlA, I nostri morti in Napoli ed in Sicilia. Statistica politica, Naples, s.n., 1860 ; ID., I primi quattro martiri della libertà italiana dell'anno 1821, Naples, s.n., 1861.

100. Carlo MASSINISSA PRESTERA, Le origini e i destini dell'umanità. I martiri di Calabria ed altri canti, Naples, Camagna, 1861. Les Martiri di Cosenza de Biagio Miraglia sont republiés à Naples en 1864.

101. Marc MONNIER, L'Italie est-elle la terre des morts?, Paris, Hachette, 1860. 
martyrologe par la faction adverse l'illustre : les légitimistes défendant les Bourbons exilés créent le leur dès les lendemains de l’Unité italienne, déplorant les affres de la guerre civile, les crimes des révolutionnaires et les épreuves de $1^{\text {'exil }}{ }^{102}$.

Pierre-Marie Delpu Aix-Marseille Université, Faculté ALLSH 29 avenue Robert Schuman 13621 Aix-en-Provence pierre-marie.DELPU@univ-amu.fr

102. Voir notamment Simon SARLIN, Le légitimisme en armes. Histoire d'une mobilisation internationale contre l'Unité italienne, Rome, École française de Rome, 2013, p. 106-112, et surtout Carmine PINTO, «La nazione mancata. Patria, guerra civile e resistenza negli scritti dei veterani borbonici del 1860-61 », in Maria Pia CAsalena (éd.), Antirisorgimento. Appropriazioni, critiche, delegittimazioni, Bologne, Pendragon, 2013, p. 87125. 


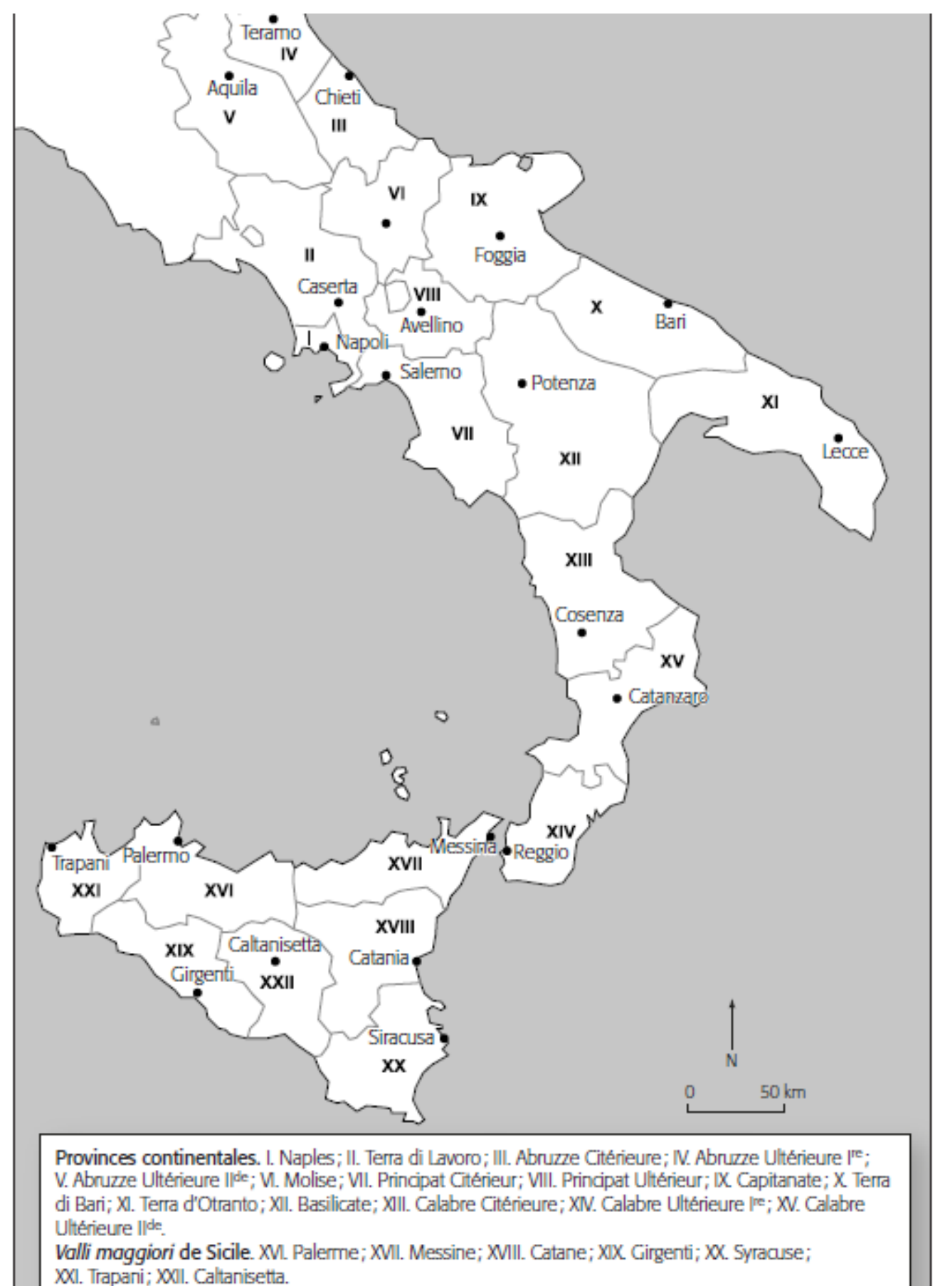

Carte 2. Les provinces administratives du royaume des Deux-Siciles (1816-1860), d'après Biagio Marzolla, Atlante corografico, storico e statistico, op. cit. 